\title{
Coordination Copolymerization of Severely Encumbered Isoalkenes with Ethylene: Enhanced Enchainment Mediated by Binuclear Catalysts and Cocatalysts
}

\author{
Hongbo Li, Liting Li, David J. Schwartz, Matthew V. Metz, and Tobin J. Marks* \\ Department of Chemistry, Northwestern University \\ Evanston, Illinois 60208-3113 \\ Louise Liable-Sands and Arnold L. Rheingold \\ Department of Chemistry and Biochemistry \\ University of California, San Diego La Jolla, CA 92093-0332
}

\section{Experimental Section}

Materials and Methods. All manipulations of air-sensitive materials were performed with rigorous exclusion of oxygen and moisture in flamed Schlenk-type glassware on a dual-manifold Schlenk line or interfaced to a high-vacuum line $\left(10^{-5}\right.$ Torr $)$, or in a nitrogen-filled Vacuum Atmospheres glove box with a high capacity recirculator $(<1$ ppm $\mathrm{O}_{2}$ ). Argon, ethylene (Matheson, polymerization grade) and isobutene (Aldrich) were purified by passage through a supported $\mathrm{MnO}$ oxygen-removal column and an activated Davison 4A molecular sieve column. Ether solvents were purified by distillation from $\mathrm{Na} / \mathrm{K}$ alloy/benzophenone ketyl. Hydrocarbon solvents were distilled under nitrogen from $\mathrm{Na} / \mathrm{K}$ alloy. All solvents for high-vacuum line manipulations were stored in vacuo over $\mathrm{Na} / \mathrm{K}$ alloy in Teflon-valve sealed bulbs. Deuterated solvents were obtained from Cambridge Isotope Laboratories (all $\geq 99$ atom $\% \mathrm{D})$, were freeze-pump- 
thaw degassed, dried over $\mathrm{Na} / \mathrm{K}$ alloy, and were stored in resealable flasks. Other nonhalogenated solvents were dried over $\mathrm{Na} / \mathrm{K}$ alloy, and halogenated solvents were distilled from $\mathrm{P}_{2} \mathrm{O}_{5}$ and stored over activated Davison $4 \mathrm{~A}$ molecular sieves. The reagents $\operatorname{Ti}\left(\mathrm{NMe}_{2}\right)_{4}, \mathrm{BBr}_{3}$, indene, $\mathrm{CH}_{3} \mathrm{CH}_{2} \mathrm{I}, \mathrm{Me}_{2} \mathrm{SiCl}_{2}$, 1,2-bis(3-indenyl)ethane (EBI), $\mathrm{Bu}^{\mathrm{t}} \mathrm{NH}_{2}$, and ${ }^{\mathrm{n}} \mathrm{BuLi}$ (1.6 M in hexanes) were purchased from Aldrich and used as received. The comonomers methylenecyclopentane, methylenecyclohexane, and 2-methyl-2-butene (Aldrich) were dried over $\mathrm{Na} / \mathrm{K}$ alloy. The reagents $1,4-\mathrm{Me}_{3} \mathrm{SnC}_{6} \mathrm{~F}_{4} \mathrm{SnMe}_{3}, \mathrm{~B}\left(\mathrm{C}_{6} \mathrm{~F}_{5}\right)_{3}$ (BN), (1-Me $\left.\mathrm{SiNH}^{\mathrm{t}} \mathrm{Bu}\right)\left(3\right.$-ethyl)indene, $\left(\mu-\mathrm{CH}_{2} \mathrm{CH}_{2}-3,3^{\prime}\right)\left[1-\left(\mathrm{Me}_{2} \mathrm{SiNH}^{t} \mathrm{Bu}\right) \text { indene }\right]_{2}$, and ( $\left.\mu-\mathrm{CH}_{2} \mathrm{CH}_{2}-3,3^{\prime}\right)\left[1-\left(\mathrm{Me}_{2} \mathrm{SiCl}\right) \text { indene }\right]_{2}$ were prepared and purified according to literature procedures. ${ }^{1,2}$

Physical and Analytical Measurements. NMR spectra were recorded on either Varian VXR 300 (FT $300 \mathrm{MHz},{ }^{1} \mathrm{H} ; 75 \mathrm{MHz},{ }^{13} \mathrm{C}$ ), VXR $400\left(\right.$ FT $400 \mathrm{MHz},{ }^{1} \mathrm{H} ; 100$ $\mathrm{MHz},{ }^{13} \mathrm{C} ; 377 \mathrm{MHz},{ }^{19} \mathrm{~F}$ ) or Gemini-300 (FT $300 \mathrm{MHz},{ }^{1} \mathrm{H} ; 75 \mathrm{MHz},{ }^{13} \mathrm{C} ; 282 \mathrm{MHz}$, ${ }^{19} \mathrm{~F}$ ) instruments. Chemical shifts for ${ }^{1} \mathrm{H}$ and ${ }^{13} \mathrm{C}$ spectra were referenced using internal solvent resonances and are reported relative to tetramethylsilane. ${ }^{19} \mathrm{~F}$ NMR spectra were referenced to external $\mathrm{CFCl}_{3}$. NMR experiments on air-sensitive samples were conducted in Teflon valve-sealed sample tubes (J. Young). Elemental analyses were performed by Oneida Research Services, Inc. Whitesboro, New York or by Midwest Microlab, Indianapolis, Indiana. ${ }^{13} \mathrm{C}$ NMR assays of polymer microstructure were conducted in a mixture (1:1 in volume) of 1,2,4-trichlorobezene (containing $0.1 \mathrm{M} \mathrm{Cr}(\mathrm{acac})_{3}$ solution) and 1,1,2,2-tetrachloroethane- $\mathrm{d}_{2}$ at $120{ }^{\circ} \mathrm{C}$. Signals were assigned according to the literature for ethylene + olefin copolymer and model compounds. ${ }^{3}$ 
Gel Permeation Chromatography (GPC) analysis was carried out on a Waters Alliance GPCV 2000 high temperature instrument equipped with three Polymer Laboratories $10 \mu \mathrm{m}$ Mixed B columns operating at $145^{\circ} \mathrm{C}$, and a refractive index detector. A flow rate of $1.0 \mathrm{~mL} / \mathrm{min}$. was used, and HPLC grade 1,2,4-trichlorobenzene was employed as the eluent. Typically $10 \pm 1.0 \mathrm{mg}$ of the sample was dissolved in 7.0 $\mathrm{mL}$ of TCB. The hot solutions were filtered using a $0.5 \mu \mathrm{m}$ stainless steel filter. A polystyrene universal calibration was carried out using narrow molecular weight distribution polystyrene standards from Polymer Laboratories with Ionol (4-(2,6,6trimethyl-2-cyclohexen-1-yl)-3-buten-2-ol) added as the flow marker. ${ }^{4}$

Differential Scanning Calorimetry (DSC) was carried out to measure Polymer glass transition temperatures on a TA Instruments 2920 Modulated Differential Scanning Calorimeter. Typically, ca. $8 \mathrm{mg}$ samples were examined, and a ramp rate of $10^{\circ} \mathrm{C} / \mathrm{min}$ was used to measure the polymer melting point. To erase thermal history effects, all samples were run through at least two melt-freeze cycles. The data from the second meltfreeze cycle are presented here.

Synthesis of EBICGC[Ti( $\left.\left.\mathrm{NMe}_{2}\right)_{2}\right]_{2} \mathbf{( 1 )}$. The reagent $\left(\mu-\mathrm{CH}_{2} \mathrm{CH}_{2}-3,3^{\prime}\right)[1-$ $\left(\mathrm{Me}_{2} \mathrm{SiNH}^{\mathrm{t}} \mathrm{Bu}\right)$ indene $]_{2}{ }^{1}(5.76 \mathrm{~g}, 11.2 \mathrm{mmol})$ was dissolved with $35 \mathrm{~mL}$ pentane in a 250 $\mathrm{mL}$ reaction flask. A solution of $\mathrm{Ti}\left(\mathrm{NMe}_{2}\right)_{4}(5.0 \mathrm{~g}, 22.3 \mathrm{mmol})$ in $100 \mathrm{~mL}$ toluene was then added. The resulting solution was refluxed at $110^{\circ} \mathrm{C}$ for $30 \mathrm{~h}$ with slow but constant $\mathrm{N}_{2}$ purge to remove evolved $\mathrm{HNMe}_{2}$. The concentrated solution was then cooled slowly to $0{ }^{\circ} \mathrm{C}$ to yield red crystals. The product was purified by recrystallization from toluene and washing with pentane. Yield, $4.3 \mathrm{~g}(49 \%)$. Spectroscopic and analytical data for $\mathbf{1}$ are as follows. ${ }^{1} \mathrm{H}$ NMR $\left(\mathrm{C}_{6} \mathrm{D}_{6}, 23{ }^{\circ} \mathrm{C}, 299.903 \mathrm{MHz}\right): \delta 7.906\left(\mathrm{~d}, 2 \mathrm{H},{ }^{3} \mathrm{~J}_{\mathrm{H}-\mathrm{H}}=8.7 \mathrm{~Hz}\right.$, Ind, 
$\left.\mathrm{C}_{6} \mathrm{H}_{4}\right), 7.585\left(\mathrm{~d}, 2 \mathrm{H},{ }^{3} \mathrm{~J}_{\mathrm{H}-\mathrm{H}}=8.0 \mathrm{~Hz}, \mathrm{Ind}, \mathrm{C}_{6} \mathrm{H}_{4}\right), 7.020\left(\mathrm{dd}, 2 \mathrm{H},{ }^{3} \mathrm{~J}_{\mathrm{H}-\mathrm{H}}=7.6 \mathrm{~Hz},{ }^{3} \mathrm{~J}_{\mathrm{H}-\mathrm{H}}=\right.$ $7.0 \mathrm{~Hz}$, Ind, $\left.\mathrm{C}_{6} \mathrm{H}_{4}\right), 6.905\left(\mathrm{dd}, 2 \mathrm{H},{ }^{3} \mathrm{~J}_{\mathrm{H}-\mathrm{H}}=8.4 \mathrm{~Hz},{ }^{3} \mathrm{~J}_{\mathrm{H}-\mathrm{H}}=6.6 \mathrm{~Hz}\right.$, Ind, $\left.\mathrm{C}_{6} \mathrm{H}_{4}\right), 6.333(\mathrm{~s}, 2$ H, Ind, $\mathrm{C}_{5} \mathrm{H}$ ), 3.450-3.364 (m, $4 \mathrm{H}, \mathrm{CH}_{2} \mathrm{CH}_{2}$ ), 3.027 (s, $12 \mathrm{H}, \mathrm{TiNMe}_{2}$ ), 2.350 (s, $12 \mathrm{H}$, $\mathrm{TiNMe}_{2}$ ), 1.240 (s, $18 \mathrm{H}, \mathrm{NCMe}_{3}$ ), 0.852 (s, $6 \mathrm{H}, \mathrm{SiMe}_{2}$ ), 0.643 (s, $\left.6 \mathrm{H}, \mathrm{SiMe}_{2}\right) \cdot{ }^{13} \mathrm{C}$ $\operatorname{NMR}\left(\mathrm{C}_{6} \mathrm{D}_{6}, 23{ }^{\circ} \mathrm{C}, 75.416 \mathrm{MHz}\right): \delta 133.471$ (Ind), 131.171 (Ind), 126.160 (Ind), 126.026 (Ind), 124.240 (Ind), 123.810 (Ind), 122.021 (Ind), 121.571 (Ind), 91.058 (Ind), $60.428\left(\mathrm{NCMe}_{3}\right), 49.526\left(\mathrm{TiNMe}_{2}\right), 47.816\left(\mathrm{TiNMe}_{2}\right), 34.204\left(\mathrm{NCMe}_{3}\right), 30.222$ $\left(\mathrm{CH}_{2} \mathrm{CH}_{2}\right), 5.155\left(\mathrm{SiMe}_{2}\right), 2.998\left(\mathrm{SiMe}_{2}\right)$. Anal. Calcd for $\mathrm{C}_{40} \mathrm{H}_{68} \mathrm{~N}_{6} \mathrm{Si}_{2} \mathrm{Ti}_{2}: \mathrm{C}, 61.20 ; \mathrm{H}$, 8.73; N, 10.71. Found: C, 61.41; H, 8.60; N, 10.71.

\section{Synthesis of EBICGC(TiMe $)_{2}\left(\mathbf{T i}_{2}\right)$. EBICGC[Ti(NMe $\left.)_{2}\right]_{2}(\mathbf{1})(900 \mathrm{mg}, 1.15$}

mmol) was dissolved with $100 \mathrm{~mL}$ toluene in a $250 \mathrm{~mL}$ reaction flask. A solution of $\mathrm{AlMe}_{3}(4.6 \mathrm{~mL}, 2.0 \mathrm{M}$ in hexanes) was added slowly by syringe at room temperature with stirring. The solution first turned deep red and then cloudy during the addition. The reaction mixture was then stirred at room temperature for two days. All the volatiles were next removed in vacuum, and the yellow solid product was purified by washing with pentane at room temperature. Yield, $550 \mathrm{mg}(72 \%)$. Spectroscopic and analytical data for $\mathbf{T i}_{2}$ are as follows. ${ }^{1} \mathrm{H}$ NMR $\left(\mathrm{C}_{6} \mathrm{D}_{6}, 23{ }^{\circ} \mathrm{C}, 299.903 \mathrm{MHz}\right): \delta 7.492\left(\mathrm{~d}, 2 \mathrm{H},{ }^{3} \mathrm{~J}_{\mathrm{H}-\mathrm{H}}=8.7 \mathrm{~Hz}\right.$, Ind, $\left.\mathrm{C}_{6} \mathrm{H}_{4}\right), 7.463\left(\mathrm{~d}, 2 \mathrm{H},{ }^{3} \mathrm{~J}_{\mathrm{H}-\mathrm{H}}=8.7 \mathrm{~Hz}\right.$, Ind, $\left.\mathrm{C}_{6} \mathrm{H}_{4}\right), 7.115-7.066\left(\mathrm{~m}, 2 \mathrm{H}\right.$, Ind, $\left.\mathrm{C}_{6} \mathrm{H}_{4}\right)$, 6.928 (m, 2 H, Ind, $\left.\mathrm{C}_{6} \mathrm{H}_{4}\right), 5.997$ (s, $\left.2 \mathrm{H}, \mathrm{Ind}, \mathrm{C}_{5} \mathrm{H}\right), 3.443-3.305$ (m, $4 \mathrm{H}, \mathrm{CH}_{2} \mathrm{CH}_{2}$ ), 1.457 (s, $18 \mathrm{H}, \mathrm{NCMe}_{3}$ ), 0.766 (s, $6 \mathrm{H}, \mathrm{SiMe}_{2}$ ), 0.569 (s, $6 \mathrm{H}, \mathrm{SiMe}_{2}$ ), 0.352 (s, $6 \mathrm{H}$, $\mathrm{TiMe}_{2}$ ), -0.111 (s, $\left.6 \mathrm{H}, \mathrm{TiMe}_{2}\right) .{ }^{13} \mathrm{C}$ NMR $\left(\mathrm{C}_{6} \mathrm{D}_{6}, 23{ }^{\circ} \mathrm{C}, 75.416 \mathrm{MHz}\right): \delta 134.264$ (Ind), 132.596 (Ind), 127.782 (Ind), 126.367 (Ind), 126.072 (Ind), 125.755 (Ind), 125.438 (Ind), 
124.073 (Ind), 90.165 (Ind), $58.623\left(\mathrm{NCMe}_{3}\right), 56.525\left(\mathrm{TiMe}_{2}\right), 56.061\left(\mathrm{TiMe}_{2}\right), 34.462$

(NCMe $), 30.120\left(\mathrm{CH}_{2} \mathrm{CH}_{2}\right), 4.010\left(\mathrm{SiMe}_{2}\right), 1.906\left(\mathrm{SiMe}_{2}\right)$. Anal. Calcd for

$\mathrm{C}_{36} \mathrm{H}_{56} \mathrm{~N}_{2} \mathrm{Si}_{2} \mathrm{Ti}_{2}: \mathrm{C}, 64.65 ; \mathrm{H}, 8.44 ; \mathrm{N}, 4.19$. Found: $\mathrm{C}, 63.65 ; \mathrm{H}, 8.38 ; \mathrm{N}, 4.10$.

\section{Synthesis of [1-Me $2 \mathrm{Si}\left(3-\mathrm{E}\right.$ thylindenyl)($\left.\left.{ }^{t} \mathrm{BuN}\right)\right] \mathrm{Ti}\left(\mathrm{NMe}_{2}\right)_{2}$. The ligand (1-}

$\left.\mathrm{Me}_{2} \mathrm{SiNH}^{\mathrm{t}} \mathrm{Bu}\right)$ (3-ethyl)indene $(6.1 \mathrm{~g}, 22.3 \mathrm{mmol})$ was dissolved with $20 \mathrm{~mL}$ pentane in a $250 \mathrm{~mL}$ reaction flask. A solution of $\mathrm{Ti}\left(\mathrm{NMe}_{2}\right)_{4}(5.0 \mathrm{~g}, 22.3 \mathrm{mmol})$ in $10 \mathrm{~mL}$ toluene was then added. The reaction solution was refluxed at $120^{\circ} \mathrm{C}$ for $24 \mathrm{~h}$ with slow but constant $\mathrm{N}_{2}$ purge to remove evolved $\mathrm{HNMe}_{2}$. All the volatiles were then removed in vacuum to yield a red oil. The unreacted starting materials $\mathrm{Ti}\left(\mathrm{NMe}_{2}\right)_{4}$ and the ligand (1$\left.\mathrm{Me}_{2} \mathrm{SiNH}^{\mathrm{t}} \mathrm{Bu}\right)\left(3\right.$-ethyl)indene were removed by distillation in vacuo at $70-80^{\circ} \mathrm{C}$.

Further distillation at higher temperature $\left(95-100^{\circ} \mathrm{C}\right)$ in vacuo did not lead to a purer product. Thus, the crude organotitanium product was used without further purification. Yield, $4.9 \mathrm{~g}(54 \%)$. Spectroscopic and analytical data for $\left[1-\mathrm{Me}_{2} \mathrm{Si}(3-\right.$ ethylindenyl) $\left.\left({ }^{\mathrm{t} B u N}\right)\right] \mathrm{Ti}\left(\mathrm{NMe}_{2}\right)_{2}$ are as follows. ${ }^{1} \mathrm{H} \mathrm{NMR}\left(\mathrm{C}_{6} \mathrm{D}_{6}, 23{ }^{\circ} \mathrm{C}, 300.075 \mathrm{MHz}\right): \delta$ $7.854\left(\mathrm{dt}, 1 \mathrm{H},{ }^{3} \mathrm{~J}_{\mathrm{H}-\mathrm{H}}=8.4 \mathrm{~Hz},{ }^{4} \mathrm{~J}_{\mathrm{H}-\mathrm{H}}={ }^{5} \mathrm{~J}_{\mathrm{H}-\mathrm{H}}=1 \mathrm{~Hz}, \mathrm{Ind}, \mathrm{C}_{6} \mathrm{H}_{4}\right), 7.461\left(\mathrm{dt}, 1 \mathrm{H},{ }^{3} \mathrm{~J}_{\mathrm{H}-\mathrm{H}}=8.4\right.$ $\mathrm{Hz},{ }^{4} \mathrm{~J}_{\mathrm{H}-\mathrm{H}}={ }^{5} \mathrm{~J}_{\mathrm{H}-\mathrm{H}}=1 \mathrm{~Hz}$, Ind, $\left.\mathrm{C}_{6} \mathrm{H}_{4}\right), 6.975-7.029\left(\mathrm{~m}, 1 \mathrm{H}, \mathrm{Ind}, \mathrm{C}_{6} \mathrm{H}_{4}\right)$, 6.836-6.889 (m, 1 H, Ind, $\mathrm{C}_{6} \mathrm{H}_{4}$ ), 6.142 (s, $1 \mathrm{H}$, Ind, $\mathrm{C}_{5} \mathrm{H}$ ), 3.033 (s, $3 \mathrm{H}, \mathrm{TiNMe}_{2}$ ), 2.333 (s, $3 \mathrm{H}, \mathrm{TiNMe}_{2}$ ), $2.800-2.880\left(\mathrm{~m}, 2 \mathrm{H}, \mathrm{CH}_{2} \mathrm{CH}_{3}\right), 1.243\left(\mathrm{~s}, 9 \mathrm{H}, \mathrm{NCMe}_{3}\right), 1.229\left(\mathrm{t}, 3 \mathrm{H},{ }^{3} \mathrm{~J}_{\mathrm{H}-\mathrm{H}}=7.8 \mathrm{~Hz}\right.$, $\mathrm{CH}_{2} \mathrm{CH}_{3}$ ), 0.829 (s, $3 \mathrm{H}, \mathrm{SiMe}$ ), 0.611 (s, $\left.3 \mathrm{H}, \mathrm{SiMe}_{2}\right) .{ }^{13} \mathrm{C} \mathrm{NMR}\left(\mathrm{C}_{6} \mathrm{D}_{6}, 23{ }^{\circ} \mathrm{C}, 75.462\right.$ MHz): $\delta 133.573$ (Ind), 130.879 (Ind), 128.388 (Ind), 125.929 (Ind), 123.948 (Ind), 123.729 (Ind), 122.201 (Ind), 121.327 (Ind), 90.244 (Ind), 60.326 ( $\mathrm{NCMe}_{3}$ ), 49.423 
(TiNMe $), 47.748\left(\mathrm{TiNMe}_{2}\right), 34.217\left(\mathrm{NCMe}_{3}\right), 21.518\left(\mathrm{CH}_{2} \mathrm{CH}_{3}\right), 15.468\left(\mathrm{CH}_{2} \mathrm{CH}_{3}\right)$, $5.156\left(\mathrm{SiMe}_{2}\right), 2.972\left(\mathrm{SiMe}_{2}\right)$.

\section{Synthesis of [1-Me $\left.{ }_{2} \mathrm{Si}(3-E t h y l i n d e n y l)\left({ }^{t} \mathrm{BuN}\right)\right] \mathrm{TiCl}_{2}$ Crude $\left[1-\mathrm{Me}_{2} \mathrm{Si}(3-\right.$}

ethylindenyl)( $\left.\left.{ }^{\mathrm{t}} \mathrm{BuN}\right)\right] \mathrm{Ti}\left(\mathrm{NMe}_{2}\right)_{2}(2.5 \mathrm{~g}, 6.15 \mathrm{mmol})$ was dissolved with $50 \mathrm{~mL}$ pentane in a $250 \mathrm{~mL}$ reaction flask. A solution of $\mathrm{Me}_{3} \mathrm{SiCl}(15.0 \mathrm{~mL})$ was then added by syringe at room temperature and the solution was stirred at room temperature for additional $16 \mathrm{~h}$. All the volatiles were then removed in vacuum, and the resulting red solid product was washed with pentane at room temperature. The product was next purified by recrystallization from toluene. Yield, $1.70 \mathrm{~g}(71 \%)$. Spectroscopic and analytical data for [1-Me $2 \mathrm{Si}\left(3\right.$-ethylindenyl) $\left.\left({ }^{\mathrm{t}} \mathrm{BuN}\right)\right] \mathrm{TiCl}_{2}$ are as follows. ${ }^{1} \mathrm{H}$ NMR $\left(\mathrm{C}_{6} \mathrm{D}_{6}, 23{ }^{\circ} \mathrm{C}, 299.903\right.$ MHz): $\delta 7.583\left(\mathrm{~d}, 1 \mathrm{H},{ }^{3} \mathrm{~J}_{\mathrm{H}-\mathrm{H}}=8.7 \mathrm{~Hz}\right.$, Ind, $\left.\mathrm{C}_{6} \mathrm{H}_{4}\right), 7.321\left(\mathrm{~d}, 1 \mathrm{H},{ }^{3} \mathrm{~J}_{\mathrm{H}-\mathrm{H}}=9.0 \mathrm{~Hz}\right.$, Ind, $\left.\mathrm{C}_{6} \mathrm{H}_{4}\right), 7.079-7.130\left(\mathrm{~m}, 1 \mathrm{H}, \mathrm{Ind}, \mathrm{C}_{6} \mathrm{H}_{4}\right), 6.957-7.006\left(\mathrm{~m}, 1 \mathrm{H}, \mathrm{Ind}, \mathrm{C}_{6} \mathrm{H}_{4}\right), 6.227(\mathrm{~s}, 1 \mathrm{H}$, Ind, $\left.\mathrm{C}_{5} \mathrm{H}\right), 2.955-3.084\left(\mathrm{~m}, 1 \mathrm{H}, \mathrm{CH}_{2} \mathrm{CH}_{3}\right), 2.669-2.796\left(\mathrm{~m}, 1 \mathrm{H}, \mathrm{CH}_{2} \mathrm{CH}_{3}\right), 1.328(\mathrm{~s}, 9 \mathrm{H}$, $\mathrm{NCMe}_{3}$ ), $1.133\left(\mathrm{t}, 3 \mathrm{H},{ }^{3} \mathrm{~J}_{\mathrm{H}-\mathrm{H}}=8.1 \mathrm{~Hz}, \mathrm{CH}_{2} \mathrm{CH}_{3}\right), 0.557\left(\mathrm{~s}, 3 \mathrm{H}, \mathrm{SiMe}_{2}\right), 0.369(\mathrm{~s}, 3 \mathrm{H}$, $\left.\mathrm{SiMe}_{2}\right) .{ }^{13} \mathrm{C}$ NMR $\left(\mathrm{C}_{6} \mathrm{D}_{6}, 23{ }^{\circ} \mathrm{C}, 75.462 \mathrm{MHz}\right): \delta 140.062$ (s, C, Ind), 136.150 (s, C, Ind), 135.122 (s, C, Ind), 128.746 (dd, CH, ${ }^{1} \mathrm{~J}_{\mathrm{C}-\mathrm{H}}=162 \mathrm{~Hz},{ }^{3} \mathrm{~J}_{\mathrm{C}-\mathrm{H}}=7.5 \mathrm{~Hz}$, Ind), 128.380 (dd, $\left.\mathrm{CH},{ }^{1} \mathrm{~J}_{\mathrm{C}-\mathrm{H}}=160 \mathrm{~Hz},{ }^{3} \mathrm{~J}_{\mathrm{C}-\mathrm{H}}=7.4 \mathrm{~Hz}, \mathrm{Ind}\right), 128.029\left(\mathrm{dd}, \mathrm{CH},{ }^{1} \mathrm{~J}_{\mathrm{C}-\mathrm{H}}=161 \mathrm{~Hz},{ }^{3} \mathrm{~J}_{\mathrm{C}-\mathrm{H}}=7.5 \mathrm{~Hz}\right.$, Ind), $126.564\left(\mathrm{dd}, \mathrm{CH},{ }^{1} \mathrm{~J}_{\mathrm{C}-\mathrm{H}}=166 \mathrm{~Hz},{ }^{3} \mathrm{~J}_{\mathrm{C}-\mathrm{H}}=7.5 \mathrm{~Hz}\right.$, Ind), $124.791\left(\mathrm{dd}, \mathrm{CH},{ }^{1} \mathrm{~J}_{\mathrm{C}-\mathrm{H}}=165\right.$ $\mathrm{Hz},{ }^{3} \mathrm{~J}_{\mathrm{C}-\mathrm{H}}=7.4 \mathrm{~Hz}$, Ind), 96.556 (s, C, Ind), 62.776 (m, NCMe 3 ), 32.486 (q, ${ }^{1} \mathrm{~J}_{\mathrm{C}-\mathrm{H}}=126$ $\mathrm{Hz}, \mathrm{NCMe}_{3}$ ), 22.577 (tq, ${ }^{1} \mathrm{~J}_{\mathrm{C}-\mathrm{H}}=129 \mathrm{~Hz},{ }^{3} \mathrm{~J}_{\mathrm{C}-\mathrm{H}}=5 \mathrm{~Hz}, \mathrm{CH}_{2} \mathrm{CH}_{3}$ ), $14.019\left(\mathrm{qt},{ }^{1} \mathrm{~J}_{\mathrm{C}-\mathrm{H}}=127\right.$ $\left.\mathrm{Hz},{ }^{3} \mathrm{~J}_{\mathrm{C}-\mathrm{H}}=5 \mathrm{~Hz}, \mathrm{CH}_{2} \mathrm{CH}_{3}\right), 3.238\left(\mathrm{q},{ }^{1} \mathrm{~J}_{\mathrm{C}-\mathrm{H}}=119 \mathrm{~Hz}, \mathrm{SiMe}_{2}\right), 0.915\left(\mathrm{q},{ }^{1} \mathrm{~J}_{\mathrm{C}-\mathrm{H}}=120 \mathrm{~Hz}\right.$, 
$\mathrm{SiMe}_{2}$ ). Anal. Calcd for $\mathrm{C}_{17} \mathrm{H}_{25} \mathrm{Cl}_{2} \mathrm{NSiTi}$ : C, 52.32; H, 6.45; N, 3.59. Found: C, 52.01; H, 6.38; N, 3.63.

\section{Synthesis of [1-Me $\left.2 \mathrm{Si}(3-E t h y l i n d e n y l)\left({ }^{\mathrm{t}} \mathrm{BuN}\right)\right] \mathrm{TiMe}_{2}\left(\mathrm{Ti}_{1}\right) \cdot\left[1-\mathrm{Me}_{2} \mathrm{Si}(3-\right.$}

ethylindenyl $\left.)\left({ }^{\mathrm{t}} \mathrm{BuN}\right)\right] \mathrm{TiCl}_{2}(270 \mathrm{mg}, 0.72 \mathrm{mmol})$ was dissolved with $30 \mathrm{~mL}$ ethyl ether in a $100 \mathrm{~mL}$ reaction flask. A solution of MeLi (1.03 mL, $1.44 \mathrm{M}$ in ethyl ether) was added by syringe at room temperature. The red solution turned yellow and cloudy immediately after the addition. The solution was stirred at room temperature for another $2 \mathrm{~h}$. All the volatiles were then removed in vacuum, and the yellow solid product was washed with pentane at room temperature. The product was purified by recrystallization from pentane. Yield, $178 \mathrm{mg}$ (78\%). Spectroscopic and analytical data for $\mathbf{T i}_{\mathbf{1}}$ are as follows. ${ }^{1} \mathrm{H}$ NMR $\left(\mathrm{C}_{6} \mathrm{D}_{6}, 23{ }^{\circ} \mathrm{C}, 299.903 \mathrm{MHz}\right): \delta 7.480\left(\mathrm{~d}, 1 \mathrm{H},{ }^{3} \mathrm{~J}_{\mathrm{H}-\mathrm{H}}=8.7 \mathrm{~Hz}, \mathrm{Ind}, \mathrm{C}_{6} \mathrm{H}_{4}\right), 7.451(\mathrm{~d}, 1 \mathrm{H}$, ${ }^{3} \mathrm{~J}_{\mathrm{H}-\mathrm{H}}=8.7 \mathrm{~Hz}$, Ind, $\left.\mathrm{C}_{6} \mathrm{H}_{4}\right), 7.120\left(\mathrm{dd}, 1 \mathrm{H},{ }^{3} \mathrm{~J}_{\mathrm{H}-\mathrm{H}}=8.7 \mathrm{~Hz},{ }^{3} \mathrm{~J}_{\mathrm{H}-\mathrm{H}}=8.1 \mathrm{~Hz}\right.$, Ind, $\left.\mathrm{C}_{6} \mathrm{H}_{4}\right)$, $6.918\left(\mathrm{dd}, 1 \mathrm{H},{ }^{3} \mathrm{~J}_{\mathrm{H}-\mathrm{H}}=7.2 \mathrm{~Hz},{ }^{3} \mathrm{~J}_{\mathrm{H}-\mathrm{H}}=8.1 \mathrm{~Hz}\right.$, Ind, $\left.\mathrm{C}_{6} \mathrm{H}_{4}\right), 5.951\left(\mathrm{~s}, 1 \mathrm{H}\right.$, Ind, $\left.\mathrm{C}_{5} \mathrm{H}\right), 2.706-$ $2.894\left(\mathrm{~m}, 2 \mathrm{H}, \mathrm{CH}_{2} \mathrm{CH}_{3}\right), 1.472\left(\mathrm{~s}, 9 \mathrm{H}, \mathrm{NCMe}_{3}\right), 1.223\left(\mathrm{t}, 3 \mathrm{H},{ }^{3} \mathrm{~J}_{\mathrm{H}-\mathrm{H}}=7.4 \mathrm{~Hz}, \mathrm{CH}_{2} \mathrm{CH}_{3}\right)$, $0.737(\mathrm{~s}, 3 \mathrm{H}, \mathrm{Ti}-\mathrm{Me}), 0.581\left(\mathrm{~s}, 3 \mathrm{H}, \mathrm{SiMe}_{2},{ }^{2} \mathrm{~J}_{\mathrm{Si}-\mathrm{H}}=6.8 \mathrm{~Hz}\right), 0.403\left(\mathrm{~s}, 3 \mathrm{H}, \mathrm{SiMe}_{2},{ }^{2} \mathrm{~J}_{\mathrm{Si}-\mathrm{H}}=\right.$ $6.9 \mathrm{~Hz}),-0.132$ (s, $3 \mathrm{H}, \mathrm{Ti}-\mathrm{Me}) .{ }^{13} \mathrm{C} \mathrm{NMR}\left(\mathrm{C}_{6} \mathrm{D}_{6}, 23{ }^{\circ} \mathrm{C}, 75.462 \mathrm{MHz}\right): \delta 134.362(\mathrm{C}$, Ind), 132.462 (C, Ind), 131.350 (C, Ind), 128.084 (CH, Ind), 125.565 (CH, Ind), 125.368 (CH, Ind), 125.143 (CH, Ind), 124.115 (CH, Ind), 89.631 (C, Ind), $58.539\left(\mathrm{NCMe}_{3}\right)$, 56.175 (Ti-Me), 55.414 (Ti-Me), $34.485\left(\mathrm{NCMe}_{3}\right), 21.873\left(\mathrm{CH}_{2} \mathrm{CH}_{3}\right), 14.709\left(\mathrm{CH}_{2} \mathrm{CH}_{3}\right)$, $4.082\left(\mathrm{SiMe}_{2}\right), 1.985\left(\mathrm{SiMe}_{2}\right)$. Anal. Calcd for $\mathrm{C}_{19} \mathrm{H}_{31} \mathrm{NSiTi}: \mathrm{C}, 65.31 ; \mathrm{H}, 8.94 ; \mathrm{N}, 4.01$.

Found: C, 64.77; H, 8.83; N, 3.93. 


\section{Synthesis of [1-Me $\left.\left.\mathrm{Mi}_{2} \mathrm{Si}-\mathrm{Ethylindenyl}\right)\left({ }^{t} \mathrm{BuN}\right)\right] \mathrm{TiMe}^{+} \mathrm{MeB}\left(\mathrm{C}_{6} \mathrm{~F}_{5}\right)_{3}{ }^{-}$. [1-Me ${ }_{2} \mathrm{Si}(3-$}

ethylindenyl)( $\left.\left.{ }^{\mathrm{B} u N}\right)\right] \mathrm{TiMe}_{2}\left(\mathbf{T i}_{\mathbf{1}}\right)(105 \mathrm{mg}, 0.30 \mathrm{mmol})$ and $\mathrm{B}\left(\mathrm{C}_{6} \mathrm{~F}_{5}\right)_{3}(153 \mathrm{mg}, 0.30$

mmol) were loaded into a $25 \mathrm{~mL}$ reaction flask in the glove box. On the high vacuum line, $\mathrm{C}_{6} \mathrm{H}_{6}(15 \mathrm{~mL})$ was condensed in at $-78^{\circ} \mathrm{C}$. The solution was then stirred at room temperature for $1 \mathrm{~h}$, and all volatiles were removed under high vacuum to give a deep orange solid. Pentane was next condensed in to wash the solid twice, and the yellow solid was dried under vacuum ( $10^{-5}$ Torr) for $4 \mathrm{~h}$ at room temperature. Yield, $130 \mathrm{mg}(50 \%)$. Spectroscopic and analytical data for the two isomers of this product are as follows. ${ }^{1} \mathrm{H}$ $\operatorname{NMR}\left(\mathrm{C}_{6} \mathrm{D}_{6}, 23{ }^{\circ} \mathrm{C}, 299.903 \mathrm{MHz}\right): \delta 7.16\left(\mathrm{~d}, 1 \mathrm{H},{ }^{3} \mathrm{~J}_{\mathrm{H}-\mathrm{H}}=8.7 \mathrm{~Hz}\right.$, Ind, $\left.\mathrm{C}_{6} \mathrm{H}_{4}\right), 7.09(\mathrm{~d}, 1$ $\mathrm{H},{ }^{3} \mathrm{~J}_{\mathrm{H}-\mathrm{H}}=8.7 \mathrm{~Hz}$, Ind, $\left.\mathrm{C}_{6} \mathrm{H}_{4}\right), 7.01\left(\mathrm{~d}, 1 \mathrm{H},{ }^{3} \mathrm{~J}_{\mathrm{H}-\mathrm{H}}=8.7 \mathrm{~Hz}, \mathrm{Ind}, \mathrm{C}_{6} \mathrm{H}_{4}\right), 6.99\left(\mathrm{~d}, 1 \mathrm{H},{ }^{3} \mathrm{~J}_{\mathrm{H}-\mathrm{H}}\right.$ $=8.6 \mathrm{~Hz}$, Ind, $\left.\mathrm{C}_{6} \mathrm{H}_{4}\right), 6.95\left(\mathrm{t}, 1 \mathrm{H},{ }^{3} \mathrm{~J}_{\mathrm{H}-\mathrm{H}}=7.9 \mathrm{~Hz}\right.$, Ind, $\left.\mathrm{C}_{6} \mathrm{H}_{4}\right), 6.68\left(\mathrm{t}, 1 \mathrm{H},{ }^{3} \mathrm{~J}_{\mathrm{H}-\mathrm{H}}=8.2 \mathrm{~Hz}\right.$, Ind, $\left.\mathrm{C}_{6} \mathrm{H}_{4}\right), 6.51\left(\mathrm{t}, 1 \mathrm{H},{ }^{3} \mathrm{~J}_{\mathrm{H}-\mathrm{H}}=7.5 \mathrm{~Hz}\right.$, Ind, $\left.\mathrm{C}_{6} \mathrm{H}_{4}\right), 6.19\left(\mathrm{t}, 1 \mathrm{H},{ }^{3} \mathrm{~J}_{\mathrm{H}-\mathrm{H}}=7.4 \mathrm{~Hz}\right.$, Ind, $\left.\mathrm{C}_{6} \mathrm{H}_{4}\right), 5.72\left(\mathrm{~s}, 1 \mathrm{H}, \mathrm{Ind}, \mathrm{C}_{5} \mathrm{H}\right), 5.56\left(\mathrm{~s}, 1 \mathrm{H}, \mathrm{Ind}, \mathrm{C}_{5} \mathrm{H}\right), 2.66$ - $2.47\left(\mathrm{~m}, 2 \mathrm{H}, \mathrm{CH}_{2} \mathrm{CH}_{3}\right)$, $2.17-2.05$ (m, $2 \mathrm{H}, \mathrm{CH}_{2} \mathrm{CH}_{3}$ ), 1.26 (s, $\left.3 \mathrm{H}, \mathrm{Ti}-\mathrm{Me}\right), 0.97$ (t, $3 \mathrm{H},{ }^{3} \mathrm{~J}_{\mathrm{H}-\mathrm{H}}=7.8 \mathrm{~Hz}$, $\left.\mathrm{CH}_{2} \mathrm{CH}_{3}\right), 0.89$ (s, $\left.9 \mathrm{H}, \mathrm{NCMe}_{3}\right), 0.87$ (s, $\left.3 \mathrm{H}, \mathrm{Ti}-\mathrm{Me}\right), 0.73$ (t, $3 \mathrm{H},{ }^{3} \mathrm{~J}_{\mathrm{H}-\mathrm{H}}=7.7 \mathrm{~Hz}$, $\mathrm{CH}_{2} \mathrm{CH}_{3}$ ), 0.35 (s, $\mathrm{SiMe}_{2}$ ), 0.29 (s, $\mathrm{SiMe}_{2}$ ), 0.27 (s, $\mathrm{SiMe}_{2}$ ), 0.10 (s, $\mathrm{SiMe}_{2}$ ), 0.11 (br, BMe). ${ }^{19} \mathrm{~F}$ NMR $\left(\mathrm{C}_{6} \mathrm{D}_{6}, 23{ }^{\circ} \mathrm{C}, 282.330 \mathrm{MHz}\right)$ : Isomer 1: $\quad-133.93\left(\mathrm{~d},{ }^{3} \mathrm{~J}_{\mathrm{F}-\mathrm{F}}=21 \mathrm{~Hz}, 6 \mathrm{~F}\right.$, $\left.o-\mathrm{F}, \mathrm{C}_{6} \mathrm{~F}_{5}\right),-159.70\left(\mathrm{t},{ }^{3} \mathrm{~J}_{\mathrm{F}-\mathrm{F}}=22 \mathrm{~Hz}, 3 \mathrm{~F}, p-\mathrm{F}, \mathrm{C}_{6} \mathrm{~F}_{5}\right),-164.82\left(\mathrm{~m}, 6 \mathrm{~F}, m-\mathrm{F}, \mathrm{C}_{6} \mathrm{~F}_{5}\right)$; Isomer 2: $\quad-133.62\left(\mathrm{~d},{ }^{3} \mathrm{~J}_{\mathrm{F}-\mathrm{F}}=22 \mathrm{~Hz}, 6 \mathrm{~F}, o-\mathrm{F}, \mathrm{C}_{6} \mathrm{~F}_{5}\right),-159.18\left(\mathrm{t},{ }^{3} \mathrm{~J}_{\mathrm{F}-\mathrm{F}}=20 \mathrm{~Hz}, 3 \mathrm{~F}, p-\mathrm{F}, \mathrm{C}_{6} \mathrm{~F}_{5}\right)$, 164.39 (m, 6 F, m-F, $\mathrm{C}_{6} \mathrm{~F}_{5}$ ). Anal. Calcd for $\mathrm{C}_{37} \mathrm{H}_{31} \mathrm{BF}_{15} \mathrm{NSiTi}$ C, 51.69; H, 3.63; N, 1.63. Found: C, 51.67; H, 3.67; N, 1.57 . 
Synthesis of MBICGC[Ti(NMe $\left.)_{2}\right]_{2}$. MBICGCH $2,\left(\mu-\mathrm{CH}_{2}-3,3^{\prime}\right)[1-$

$\left(\mathrm{Me}_{2} \mathrm{SiNH}^{\mathrm{t}} \mathrm{Bu} \text { )indenyl }\right]_{2}$, (prepared by the procedure outlined in reference 2, $5.00 \mathrm{~g}, 9.96$ mmol) was dissolved in $35 \mathrm{~mL}$ pentane. A solution of $\mathrm{Ti}\left(\mathrm{NMe}_{2}\right)_{4}(4.46 \mathrm{~g}, 19.9 \mathrm{mmol})$ in $100 \mathrm{~mL}$ toluene was then added, and the solution refluxed at $110^{\circ} \mathrm{C}$ for $48 \mathrm{~h}$ under slow but constant $\mathrm{N}_{2}$ purge to remove evolved $\mathrm{HNMe}_{2}$. The product consists of two diastereomers, $(R S, S R)$ and $(S S, R R)(1: 1.4$ or 1.4:1 ratio), as indicated by NMR spectra. Yield, $5.1 \mathrm{~g}(66 \%)$. Spectroscopic and analytical data: ${ }^{1} \mathrm{H}$ NMR $\left(\mathrm{C}_{6} \mathrm{D}_{6}, 23^{\circ} \mathrm{C}\right): \delta 7.766-$ $7.749\left(\mathrm{~m}, 2 \mathrm{H}, \mathrm{Ind}, \mathrm{C}_{6} \mathrm{H}_{4}\right), 7.503-7.486\left(\mathrm{~m}, 2 \mathrm{H}\right.$, Ind, $\left.\mathrm{C}_{6} \mathrm{H}_{4}\right)$, 6.843-6,644 (m, $4 \mathrm{H}$, Ind, $\left.\mathrm{C}_{6} \mathrm{H}_{4}\right), 6.554\left(\mathrm{~s}, 2 \mathrm{H}\right.$, Ind, $\left.\mathrm{C}_{5} \mathrm{H}\right), 6.429\left(\mathrm{~s}, 2 \mathrm{H}\right.$, Ind, $\left.\mathrm{C}_{5} \mathrm{H}\right), 4.915\left(\mathrm{br}, \mathrm{s}, 2 \mathrm{H}\right.$, bridge- $\mathrm{CH}_{2}$ ), 3.285 (s, $6 \mathrm{H}, \mathrm{TiNMe}_{2}$ ), 3.240 (s, $6 \mathrm{H}, \mathrm{TiNMe}_{2}$ ), 2.396 (s, $6 \mathrm{H}, \mathrm{TiNMe}_{2}$ ), 2.383 (s, $6 \mathrm{H}$, TiNMe 2 ), 1.269 (s, 9 H, $\mathrm{NCMe}_{3}$ ), 1.245 (s, $9 \mathrm{H}, \mathrm{NCMe}_{3}$ ), 0.802 (s, $6 \mathrm{H}, \mathrm{SiMe}$ ), 0.788 (s, $\left.6 \mathrm{H}, \mathrm{SiMe}_{2}\right), 0.624$ (s, $6 \mathrm{H}, \mathrm{SiMe}$ ), 0.612 (s, $\left.6 \mathrm{H}, \mathrm{SiMe}_{2}\right) .{ }^{13} \mathrm{C} \mathrm{NMR}\left(\mathrm{C}_{6} \mathrm{D}_{6}\right): \delta 134.075$ (Ind), 131.758 (Ind), 126.763 (Ind), 126.714 (Ind), 124.840 (Ind), 124.407 (Ind), 122.615 (Ind), 122.188 (Ind), 91.657 (Ind), $60.941\left(\mathrm{NCMe}_{3}\right), 50.188\left(\mathrm{TiNMe}_{2}\right), 48.366\left(\mathrm{TiNMe}_{2}\right)$, $34.615\left(\mathrm{NCMe}_{3}\right), 11.458\left(-\mathrm{CH}_{2}-\right), 5.342\left(\mathrm{SiMe}_{2}\right), 3.328\left(\mathrm{SiMe}_{2}\right)$.

Synthesis of MBICGC(TiMe $)_{2}\left(\mathbf{C 1}_{2}-\mathbf{T i}_{2}\right)$. MBICGC[Ti(NMe $\left.)_{2}\right]_{2}(800 \mathrm{mg}, 1.04$ mmol) was dissolved with $50 \mathrm{~mL}$ pentane. A solution of $\mathrm{AlMe}_{3}(5.0 \mathrm{~mL}, 2.0 \mathrm{M}$ in hexanes) was added slowly by syringe at $25^{\circ} \mathrm{C}$ with stirring. The solution first became yellow and then cloudy during the addition. The solution was stirred at $25^{\circ} \mathrm{C}$ for an additional $48 \mathrm{~h}$. All the volatiles were then removed in vacuo, and the white solid product was washed with pentane. Yield, $587 \mathrm{mg}(86 \%)$. Spectroscopic and analytical data for $\mathbf{C}_{\mathbf{1}}-\mathbf{T i}_{2}:{ }^{1} \mathrm{H}$ NMR $\left(\mathrm{C}_{6} \mathrm{D}_{6}, 23^{\circ} \mathrm{C}\right): \delta 7.701\left(\mathrm{~d}, 2 \mathrm{H},{ }^{3} \mathrm{~J}_{\mathrm{H}-\mathrm{H}}=8.0 \mathrm{~Hz}\right.$, Ind, $\left.\mathrm{C}_{6} \mathrm{H}_{4}\right), 7.422(\mathrm{~d}, 2$ $\mathrm{H},{ }^{3} \mathrm{~J}_{\mathrm{H}-\mathrm{H}}=7.0 \mathrm{~Hz}$, Ind, $\left.\mathrm{C}_{6} \mathrm{H}_{4}\right), 7.032\left(\mathrm{dd}, 2 \mathrm{H},{ }^{3} \mathrm{~J}_{\mathrm{H}-\mathrm{H}}=7.0 \mathrm{~Hz},{ }^{3} \mathrm{~J}_{\mathrm{H}-\mathrm{H}}=6.7 \mathrm{~Hz}\right.$, Ind, $\left.\mathrm{C}_{6} \mathrm{H}_{4}\right)$, 
$6.824\left(\mathrm{dd}, 2 \mathrm{H},{ }^{3} \mathrm{~J}_{\mathrm{H}-\mathrm{H}}=7.0 \mathrm{~Hz}, \mathrm{Ind}, \mathrm{C}_{6} \mathrm{H}_{4}\right), 6.212\left(\mathrm{~s}, 2 \mathrm{H}, \mathrm{Ind}, \mathrm{C}_{5} \mathrm{H}\right), 4.730$ (br, s, $2 \mathrm{H}$, $\mathrm{CH}_{2}$ ), 1.506 (s, $9 \mathrm{H}, \mathrm{NCMe}_{3}$ ), 0.989 (s, $6 \mathrm{H}, \mathrm{SiMe}_{2}$ ), 0.538 (s, $6 \mathrm{H}, \mathrm{SiMe}_{2}$ ), 0.349 (s, $6 \mathrm{H}$, $\mathrm{TiMe}_{2}$ ), -0.030 (s, $6 \mathrm{H}, \mathrm{TiMe}_{2}$ ). Anal. Calcd for $\mathrm{C}_{35} \mathrm{H}_{54} \mathrm{~N}_{2} \mathrm{Si}_{2} \mathrm{Ti}_{2}: \mathrm{C}, 64.20 ; \mathrm{H}, 8.31 ; \mathrm{N}$, 4.28 Found: C, 63.31; H, 8.15; N, 4.16.

Synthesis of $\mathbf{1 , 4}-\mathbf{C}_{6} \mathbf{F}_{4}\left[\mathbf{B}\left(\mathbf{C}_{6} \mathbf{F}_{5}\right)_{2}\right]_{2}$. Method I . Into a thick-walled reaction vessel filled with a J. Young valve was added $\left(\mathrm{C}_{6} \mathrm{~F}_{5}\right)_{2} \mathrm{BCl}^{5 \mathrm{a}}(2.87 \mathrm{~g}, 7.56 \mathrm{mmol})$ and 1,4$\mathrm{C}_{6} \mathrm{~F}_{4}\left(\mathrm{SnMe}_{3}\right)_{2}{ }^{5 \mathrm{~b}}(0.60 \mathrm{~g}, 1.26 \mathrm{mmol})$. Toluene $(40 \mathrm{~mL})$ was next added, the contents cooled to $-78^{\circ} \mathrm{C}$, the flask was evacuated to 0.1 Torr, and the J. Young valve closed. The flask was heated at $140^{\circ} \mathrm{C}$ for $72 \mathrm{~h}$ behind a blast shield. The solvent was then removed in vacuo, and the residue washed with pentane ( 4 × $20 \mathrm{~mL}$ ). The resulting light yellow solid was dried under dynamic vacuum $\left(10^{-5}\right.$ Torr $)$ for $12 \mathrm{~h}$, giving the product as a microcrystalline pale yellow solid $(0.75 \mathrm{~g}, 71 \%)$.

Method II. The reagents $1,4-\mathrm{C}_{6} \mathrm{~F}_{4}\left(\mathrm{SnMe}_{3}\right)_{2}(2.2 \mathrm{~g}, 4.6 \mathrm{mmol})$ and $\left(\mathrm{C}_{6} \mathrm{~F}_{5}\right)_{2} \mathrm{BCl}$ $(10.6 \mathrm{~g}, 27.9 \mathrm{mmol})$ were loaded in a $150 \mathrm{~mL}$ reaction flask in the glovebox. The flask was then placed in a $130^{\circ} \mathrm{C}-140^{\circ} \mathrm{C}$ bath. The starting materials melted at this temperature and the mixture was stirred overnight. At the completion of the reaction, the color of the mixture was light yellow. The mixture was cooled to room temperature to yield a wet, light yellow solid. The solid was washed with pentane ( 2 x $20 \mathrm{~mL})$, and all the volatiles then removed in vacuo to give light yellow solid. Next, all volatile byproducts were sublimed away at $70^{\circ} \mathrm{C}$ for $4 \mathrm{~h}$, and the pure product was collected as light yellow solid (2.9 g, 75\%). 
Spectroscopic and analytical data are as follows. MS (EI): m/e 838 (M+, 100\%).

${ }^{19} \mathrm{~F}$ NMR $\left(\mathrm{CD}_{2} \mathrm{Cl}_{2}, 23^{\circ} \mathrm{C}\right): \delta-125.7\left(\mathrm{br}, 8 \mathrm{~F}\right.$, ortho $\left.\mathrm{C}_{6} \mathrm{~F}_{5}\right),-128.2\left(\mathrm{br}, 4 \mathrm{~F}, \mathrm{C}_{6} \mathrm{~F}_{4}\right),-141.1(\mathrm{br}$, 4F, para $\left.\mathrm{C}_{6} \mathrm{~F}_{5}\right),-159.1\left(\mathrm{br}, 8 \mathrm{~F}\right.$, meta $\left.\mathrm{C}_{6} \mathrm{~F}_{5}\right) .{ }^{19} \mathrm{~F}$ NMR $\left(\mathrm{C}_{6} \mathrm{D}_{6}\right): \delta-128.0\left(\mathrm{~d}, 8 \mathrm{~F}\right.$, ortho $\left.\mathrm{C}_{6} \mathrm{~F}_{5}\right)$, $-130.35\left(\mathrm{~s}, 4 \mathrm{~F}, \mathrm{C}_{6} \mathrm{~F}_{4}\right),-140.1\left(\mathrm{~s}, 4 \mathrm{~F}\right.$, para $\left.\mathrm{C}_{6} \mathrm{~F}_{5}\right),-159.6\left(\mathrm{br}, 8 \mathrm{~F}\right.$, meta $\left.\mathrm{C}_{6} \mathrm{~F}_{5}\right)$. Anal. Calcd for $\mathrm{C}_{30} \mathrm{~F}_{24} \mathrm{~B}_{2}: \mathrm{C}, 43.00 ; \mathrm{H}, 0.00$. Found: $\mathrm{C}, 42.72 ; \mathrm{H}, 0.01$.

\section{Synthesis of $\left[\left(\mathrm{C}_{5} \mathbf{H}_{5}\right)_{2} \mathrm{ZrMe}^{+}\right]_{2}\left\{\mathbf{M e}_{2} \mathbf{1}, \mathbf{4}-\mathrm{C}_{6} \mathbf{F}_{4}\left[\mathbf{B}\left(\mathrm{C}_{6} \mathbf{F}_{5}\right)_{2}\right]_{2}\right\}^{2-}(3)\left(\mathrm{C}_{5} \mathrm{H}_{5}\right)_{2} \mathrm{ZrMe}_{2}$}

(251 mg, $1.00 \mathrm{mmol})$ and $1,4-\mathrm{C}_{6} \mathrm{~F}_{4}\left[\mathrm{~B}\left(\mathrm{C}_{6} \mathrm{~F}_{5}\right)_{2}\right]_{2}\left(\mathbf{B N}_{2} ; 420 \mathrm{mg}, 0.50 \mathrm{mmol}\right)$ were loaded into a $25 \mathrm{~mL}$ reaction flask in the glove box. On the high vacuum line, $\mathrm{C}_{6} \mathrm{H}_{6}(15 \mathrm{~mL})$ was condensed in at $-78^{\circ} \mathrm{C}$. The solution was then stirred at room temperature for $1 \mathrm{~h}$, and all volatiles were removed under high vacuum to give a pale yellow solid. Pentane was condensed in to wash the solid twice, and the solid was dried under vacuum $\left(10^{-5}\right.$ Torr $)$ for $4 \mathrm{~h}$ at room temperature. Yield, $415 \mathrm{mg}(62 \%) .{ }^{1} \mathrm{H} \mathrm{NMR}\left(\mathrm{CD}_{2} \mathrm{Cl}_{2}, 23^{\circ} \mathrm{C}\right): .6 .29(\mathrm{~s}$, 20H), 0.45 (br, 6H), 0.29 (br, 6H) ppm. ${ }^{19} \mathrm{~F} \mathrm{NMR}\left(\mathrm{CD}_{2} \mathrm{Cl}_{2}\right): \quad-131.5\left(\mathrm{~d},{ }^{3} \mathrm{~J}_{\mathrm{FF}}=18 \mathrm{~Hz}\right.$, $8 \mathrm{~F}$, ortho $\left.\mathrm{C}_{6} \mathrm{~F}_{5}\right),-142.8\left(\mathrm{br}, 4 \mathrm{~F}, \mathrm{C}_{6} \mathrm{~F}_{4}\right),-162.4\left(\mathrm{br}, 4 \mathrm{~F}\right.$, para $\left.\mathrm{C}_{6} \mathrm{~F}_{5}\right),-165.3(\mathrm{br}, 8 \mathrm{~F}$, meta $\left.\mathrm{C}_{6} \mathrm{~F}_{5}\right) \mathrm{ppm}$.

Crystal Structure Determination of EBICGC[Ti(NMe $\left.)_{2}\right]_{2}(\mathbf{1})$. Suitable crystals of 1 for diffraction studies were grown by slowly cooling of a concentrated toluene solution of 1 from $90^{\circ} \mathrm{C}$ to room temperature. The crystals consist of two diastereomers $(R S, S R)$ and $(S S, R R)\left(1: 1.3\right.$ or $1.3: 1$ ratio) as indicated by ${ }^{1} \mathrm{H}$ NMR spectroscopy. Crystals of one diastereomer $(R S, S R)$ were found to be suitable for X-ray diffraction. The crystal was mounted on a glass fiber and transferred to a Siemens P4 diffractometer equipped with a SMART1000 CCD detector. SMART software was used for data collection and was integrated with SAINT software. Refinement used SHELXTL (version 5.0). The highest 
symmetry found was triclinic and the centrosymmetric setting was initially assumed. This selection is supported by the results of the refinement. The structure was solved by direct methods and refined with anisotropic thermal parameters for all nonhydrogen atoms. Hydrogen atoms were placed in idealized locations. Crystallographic data are collected in Table 1.

X-Ray Crystal Structure Determinations of [1-Me 2 Si(3-Ethylindenyl)( $\left.\left.{ }^{\mathrm{B}} \mathrm{BuN}\right)\right] \mathrm{TiMe}_{2}$ ( $\left.\mathrm{Ti}_{1}\right)$, [1-Me $\left.2 \mathrm{Si}(3-\mathrm{Ethylindenyl})\left({ }^{\mathrm{t}} \mathrm{BuN}\right)\right] \mathrm{TiMe}^{+} \mathrm{MeB}\left(\mathrm{C}_{6} \mathrm{~F}_{5}\right)_{3}{ }^{-}$(2), and $\left[\left(\mathrm{C}_{5} \mathrm{H}_{5}\right)_{2} \mathrm{ZrMe}^{+}\right]_{2}\left\{\mathrm{Me}_{2} 1,4-\mathrm{C}_{6} \mathrm{~F}_{4}\left[\mathrm{~B}\left(\mathrm{C}_{6} \mathrm{~F}_{5}\right)_{2}\right]_{2}\right\}^{2-}(3)$.

Suitable crystals of $\mathbf{T i}_{\mathbf{1}}, \mathbf{3}$, and $\mathbf{4}$ for diffraction studies were grown by slowly cooling of concentrated toluene solutions from room temperature to $-30^{\circ} \mathrm{C}$. Inside the glove box, the crystals were placed on a glass slide and covered in Infineum V8512 oil. The crystals were then removed from the box, and a suitable crystal was chosen under a microscope using plane-polarized light. The crystal was mounted on a glass fiber and transferred to a Bruker SMART $1000 \mathrm{CCD}$ area detector diffractometer with a nitrogen cold-stream at $153(2) \mathrm{K}$. Twenty frames (20 second exposures, $0.3^{\circ}$ slices) were collected in three areas of space to determine the orientation matrix. The parameters for data collection were determined by the peak intensities and widths from the sixty frames used to determine the orientation matrix. The faces of the crystal were indexed and data collection was begun. After data collection, the frames were integrated, the initial crystal structure solved by direct methods, the structure solution was expanded through successive least-squares cycles, absorption corrections were applied, and the final solution was determined. 
Ethylene + Olefin Copolymerization Experiments. On the high vacuum line $\left(10^{-5}\right.$ Torr), ethylene copolymerizations were carried out in $25 \mathrm{~mL}$ flasks equipped with a magnetic stirring bar, Morton indentations, and a thermocouple probe. In a typical experiment, in the glove-box, measured amounts of catalyst and cocatalyst were loaded into a $25 \mathrm{~mL}$ flask, which was then evacuated on the vacuum line. Toluene $(10 \mathrm{~mL})$ and comonomer $(2 \mathrm{~mL})$ were next vacuum-transferred in sequence into the flask at $-78^{\circ} \mathrm{C}$. The mixture was warmed to room temperature and vigorously stirred for 2 min for activation until the solution turned to clear red. Following this procedure, the flask was then exposed to $1.0 \mathrm{~atm}$ of ethylene. The temperature of the toluene solution was monitored in representative experiments using a thermocouple (OMEGA Type K thermocouple with a Model HH21 microprocessor thermometer). The reaction exotherm temperature rise was invariably less than $5^{\circ} \mathrm{C}$ during these polymerizations. After a measured time interval (short to minimize mass transport and exotherm effects), the polymerization was quenched by the addition of $1.5 \mathrm{~mL} 2 \%$ acidified methanol. Another $50 \mathrm{~mL}$ methanol was then added and the polymer was collected by filtration, washed with methanol, and dried on the high vacuum line overnight to a constant weight.

\section{Reference}

(1) Li, L.; Metz, M. V.; Li, H.; Chen, M.-C.; Marks, T. J.; Liable-Sands, L.; Rheingold, A. L.; J. Am. Chem. Soc.; 2002; 124; 12725-12741.

(2) Li, H.; Li, L.; Marks, T.J. Liable-Sands, L.; Rheingold, A. L.; J. Am. Chem. Soc. 2003, 124; 10788-10789. 
(3) (a) Breitmaier, E.; Voelter, W. Carbon-13 NMR Spectroscopy; VCH Publishers:

Weinheim, 1987. (b) Spectral Database for Organic Compounds, SDBS. (c) The assignment is based on relative intensity changes of the these peaks at different incorporation level.

(4) Protivova, J.; Pospisil, J.; Zikmund, L. J. Polym. Sci. Polym Symposia 1973, 40 233-

43.

(5) (a) Chambers, R. D.; Chivers, T. J. Chem. Soc. 1965, 3933-9. (b) Massey, A. G.;

Park. A. J. J. Organomet. Chem. 1964, 2, 245. 